\title{
Unequal arm space-borne gravitational wave detectors
}

\author{
Shane L. Larson* \\ Space Radiation Laboratory, California Institute of Technology, Pasadena, California 91125
}

Ronald W. Hellings ${ }^{\dagger}$ and William A. Hiscock ${ }^{\ddagger}$

Department of Physics, Montana State University, Bozeman, Montana 59717

(Received 26 June 2002; published 16 September 2002)

\begin{abstract}
Unlike ground-based interferometric gravitational wave detectors, large space-based systems will not be rigid structures. When the end stations of the laser interferometer are freely flying spacecraft, the armlengths will change due to variations in the spacecraft positions along their orbital trajectories, so the precise equality of the arms that is required in a laboratory interferometer to cancel laser phase noise is not possible. However, using a method discovered by Tinto and Armstrong, a signal can be constructed in which laser phase noise exactly cancels out, even in an unequal arm interferometer. We examine the case where the ratio of the armlengths is a variable parameter, and compute the averaged gravitational wave transfer function as a function of that parameter. Example sensitivity curve calculations are presented for the expected design parameters of the proposed LISA interferometer, comparing it to a similar instrument with one arm shortened by a factor of 100 , showing how the ratio of the armlengths will affect the overall sensitivity of the instrument.
\end{abstract}

DOI: 10.1103/PhysRevD.66.062001

PACS number(s): 04.80.Nn, 95.55.Ym

\section{INTRODUCTION}

One of the differences between laboratory and space laser interferometer gravitational wave detectors is that, in the laboratory, the two arms of the interferometer that is used to detect changes in the spacetime geometry are maintained at nearly equal lengths. Therefore, when the signals from the two perpendicular arms are combined, the laser phase noise in the differenced signals cancels almost exactly. In space, a laser interferometer gravitational wave detector such as the Laser Interferometer Space Antenna (LISA) [1] will have free-flying spacecraft as the end masses, and precise equality of the arms is not possible. Other methods must then be used to eliminate laser phase noise from the system [2-4]. These methods involve a heterodyne measurement for each separate arm of the interferometer and data processing that combines data from both arms to generate a signal that is free of laser phase noise. In a previous paper ([5], hereafter called paper I), the sensitivity curves for space detectors using these techniques were generated by explicitly calculating transfer functions for signal and noise, as modified by the data processing algorithms. While the algorithms have been shown [4], in principle, to eliminate the laser phase noise in the detectors regardless of the lengths of the two arms, the transfer functions have previously only been calculated for the case of equal arms [5-8]. In this paper we extend the calculation of the noise and signal transfer functions to the case of arbitrarily chosen armlengths.

One of the goals of paper I was to provide a uniform system for evaluating the sensitivity of various configurations of space gravitational detectors. This paper extends that capability to configurations in which the armlengths are sig-

\footnotetext{
*Electronic address: shane@srl.caltech.edu

†Electronic address: hellings@physics.montana.edu

‡Electronic address: hiscock@montana.edu
}

nificantly different from each other. For example, a proposal by Bernard Schutz at the 2000 LISA Symposium in Golm, Germany [9], suggested a modification to the current LISA design in which a fourth spacecraft is inserted in the middle of one of the legs of the interferometer to produce two independent interferometers, each with one leg half the length of the other (see Fig. 1). The goal of such a design was to be able to cross-correlate the independent interferometers to search for the stochastic cosmic gravitational wave background. Using the analysis presented here, one will be able to determine the sensitivity of such an interferometer and judge the scientific value of the proposed modification.

As in paper I, the analysis begins with the response of a round-trip electromagnetic tracking signal to the passage of a

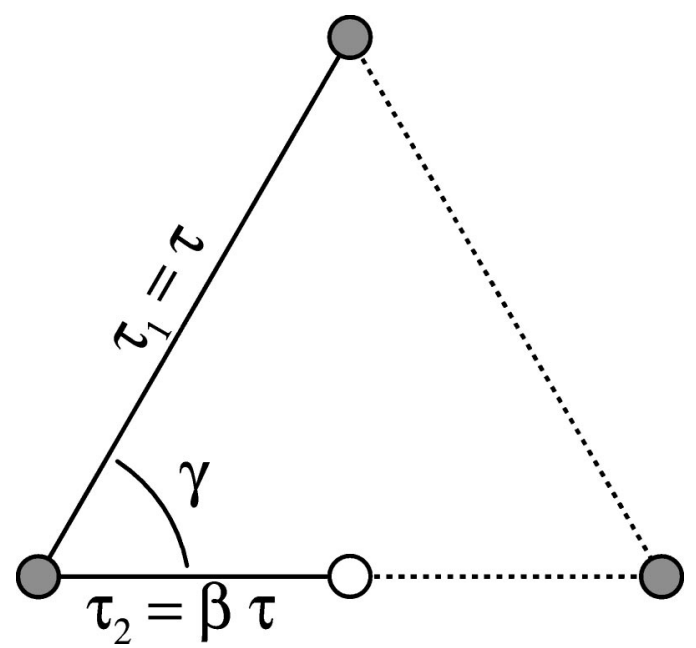

FIG. 1. An unequal arm geometry used here assumes two arms of length $\tau$ and $\beta \tau$, with an enclosed angle $\gamma$ (the interferometer opening angle). Depicted here is the nominal LISA constellation of three spacecraft in an equilateral triangle $(\beta=1)$, and a proposed extension which places a fourth spacecraft midway down one of the arms $(\beta=1 / 2)$. 
gravitational wave, as derived by Estabrook and Wahlquist [10]. A gravitational wave of amplitude $h(t)$ will produce a Doppler shift $\Delta \nu$ in the received frequency, relative to the outgoing signal with fundamental frequency $\nu_{0}(t)$. The shift is given by

$$
\begin{aligned}
\frac{\Delta \nu(t, \theta, \psi)}{\nu_{0}}= & \frac{1}{2} \cos (2 \psi)[(1-\cos \theta) h(t)+2 \cos \theta \\
& \times h(t-\tau-\tau \cos \theta)-(1+\cos \theta) h(t-2 \tau)],
\end{aligned}
$$

where $\tau$ is the one-way light travel time between spacecraft, $\theta$ is the angle between the line connecting the spacecraft and the line of sight to the source, and $\psi$ is a principal polarization angle of the quadrupole gravitational wave. It is desirable to work in frequency space, so $h(t)$ is written in terms of its Fourier transform $\tilde{h}(\omega)$. If the Doppler record is sampled for a time $T$ then $h(t)$ is related to its Fourier transform by

$$
h(t)=\frac{\sqrt{T}}{2 \pi} \int_{-\infty}^{+\infty} \widetilde{h}(\omega) e^{i \omega t} d \omega,
$$

where the $\sqrt{T}$ normalization factor is used to keep the power spectrum roughly independent of time. Using this definition of the Fourier transform, the frequency shift of Eq. (1) can be written as

$$
\begin{aligned}
\Delta \nu(t, \theta, \psi)= & \frac{\nu_{0} \sqrt{T}}{2 \pi} \int_{-\infty}^{+\infty} \frac{1}{2} \cos (2 \psi) \widetilde{h}(\omega, \theta, \phi, \psi)[(1-\mu) \\
& \left.+2 \mu e^{-i \omega \tau(1+\mu)}-(1+\mu) e^{-i 2 \omega \tau}\right] e^{i \omega t} d \omega,
\end{aligned}
$$

where $\mu \equiv \cos \theta$. The quantity that is actually read out in a laser interferometer tracking system is phase, so Eq. (3) is integrated to find the phase in cycles

$$
\Delta \phi(t, \theta, \psi)=\int \Delta \nu(t, \theta, \psi) d t .
$$

In paper I, a strain-like variable $z$ was formed by dividing the $\Delta \phi$ in Eq. (4) by $\nu_{0} \tau$ and the analysis was done using this variable. Since both arms had roughly the same length in paper I and carried nearly the same frequency, there was only a scale difference between using $\Delta \phi$ and using $z$ as the observable, and linear combinations of $z$ were the same as linear combinations of $\Delta \phi$. However, when the two armlengths are different, this is no longer the case, and one must be careful as to what is taken to be the observable for use in noise-cancelling data analysis.

In the laser phase-noise-cancellation algorithms that will be presented in Sec. II, it is relative phase and not strain that can be combined to create laser-noise-free signals. To understand how this arises, consider a case where laser signals in two arms are phase-locked to each other, with $\nu_{1}$ as the frequency of the master laser in the first arm and $\nu_{2}=\chi \nu_{1}$ as the frequency in the second arm, with $\chi$ as the ratio of the two frequencies. Then a phase noise excursion $\delta \phi_{1}$ in the first arm will produce a phase noise excursion $\chi \delta \phi_{1}$ in the second arm. Thus it will be linear combinations of $z_{i}$ $=\phi_{i} / \nu_{i}$ that will allow the two noise terms to cancel. Therefore, in this paper, the gravitational wave observable in the $i$ th arm is defined to be

$$
\begin{aligned}
z_{i}(t, \theta, \psi) \equiv & \frac{\Delta \phi_{i}(t, \theta, \psi)}{\nu_{i}} \\
= & \frac{\sqrt{T}}{4 \pi} \int_{-\infty}^{+\infty} d \omega \cos (2 \psi) \widetilde{h}(\omega)[(1-\mu) \\
& \left.+2 \mu e^{-i \omega \tau(1+\mu)}-(1+\mu) e^{-i 2 \omega \tau}\right] \frac{1}{\omega} e^{i \omega t},
\end{aligned}
$$

where Eq. (3) has been used to expand $\Delta \nu(t, \theta, \psi)$ and where arbitrary constant phases have been set to zero in the integration. It should be noted that $z_{i}$ is a different observable than the strain variable that was labeled $z_{i}$ in paper I. It should also be noted that $z_{i}$, as it is now defined, has units of time, so Eq. (5) gives the time delay in seconds produced by the passage of a gravitational wave through the detector.

\section{SENSITIVITY CURVES}

\section{A. Instrument signal}

Tinto and Armstrong [2] originally showed that the preferred signal for purposes of data analysis is not the traditional Michelson combination (difference of both arms), but rather a new combination $X(t)$, given in the time domain by [11]

$$
\begin{aligned}
X(t)= & s_{1}(t)-s_{2}(t)-s_{1}\left(t-2 \tau_{2}\right)+s_{2}\left(t-2 \tau_{1}\right) \\
= & z_{1}(t)-z_{2}(t)-z_{1}\left(t-2 \tau_{2}\right)+z_{2}\left(t-2 \tau_{1}\right) \\
& +n_{1}(t)-n_{1}\left(t-2 \tau_{2}\right)-n_{2}(t)+n_{2}\left(t-2 \tau_{1}\right),(6)
\end{aligned}
$$

where $s_{i}(t)$ is the data stream from the $i^{\text {th }}$ interferometer arm, composed of the signal $z_{i}(t)$ of interest [given by Eq. (5)] and the combined noise spectra in each of the interferometer arms, $n_{i}(t)$. The armlengths are taken to be unequal, with armlength $\tau_{i}$ in the $i^{\text {th }}$ arm. This combination is devoid of laser phase noise for all values of the two armlengths $\tau_{1}$ and $\tau_{2}$.

In order to construct this combination, the armlengths must be known to sufficient accuracy and the data samples with the correct offsets must be available. Details of this requirement are worked out by Hellings [3].

To determine the sensitivity using the $X(t)$ variable, it is necessary to establish a relationship between the amplitude of a gravitational wave incident on the detector and the size of the $X(t)$ signal put out by the instrument. The noise in the detector will limit this sensitivity, and must also be included in the analysis. The part of $X(t)$ containing the gravitational wave signal is [12] 


$$
\Lambda(t)=z_{1}(t)-z_{2}(t)-z_{1}\left(t-2 \tau_{2}\right)+z_{2}\left(t-2 \tau_{1}\right) .
$$

The transfer function $\mathcal{R}(\omega)$, which connects the spectral density of the instrument output $S_{\bar{\Lambda}}(\omega)$ with the spectral density of the gravitational waves $S_{h}(\omega)$ in frequency space, is defined via

$$
S_{\bar{\Lambda}}(\omega)=S_{h}(\omega) \mathcal{R}(\omega),
$$

where the bar over the $\Lambda$ in Eq. (8) indicates an average over source polarization and direction. The gravitational wave amplitude spectral density $S_{h}(\omega)$ is defined by

$$
S_{h}(\omega)=|\widetilde{h}(\omega)|^{2},
$$

where $\widetilde{h}(\omega)$ is the Fourier amplitude defined in Eq. (2), so that the mean-square gravitational wave strain is given by

$$
\left\langle h^{2}\right\rangle=\lim _{T \rightarrow \infty} \frac{1}{T} \int_{0}^{T} h(t)^{2} d t=\frac{1}{2 \pi} \int_{0}^{\infty} S_{h}(\omega) d \omega,
$$

where $T$ is the record length. Similarly, the instrumental response $S_{\bar{\Lambda}}(\omega)$ is defined such that

$$
\overline{\left\langle\Lambda^{2}\right\rangle}=\frac{1}{2 \pi} \int_{0}^{\infty} S_{\bar{\Lambda}}(\omega) d \omega,
$$

where the brackets indicate a time average. In the next section, the transfer function from the gravitational wave amplitude $h$ to the instrument signal $\bar{\Lambda}$ is worked out.

\section{B. Gravitational wave transfer function}

Let us take the ratio of the two armlengths in the interferometer to be an adjustable parameter, $\beta$, taking on values between 0 and 1 , such that $\tau_{1}=\tau$ and $\tau_{2}=\beta \tau$. The average power in the part of $X(t)$ which contains the gravitational wave signal is given by

$$
\left\langle\Lambda^{2}\right\rangle=\lim _{T \rightarrow \infty} \frac{1}{T} \int_{0}^{T}|\Lambda|^{2} d t
$$

where $\Lambda$ is defined by Eq. (7). Using the definition of $z$ from Eq. (5) this can be expanded to yield

$$
\left\langle\Lambda^{2}\right\rangle=\frac{1}{2 \pi} \int_{0}^{\infty} d \omega \widetilde{h}^{2}(\omega) \frac{1}{\omega^{2}}\left[T_{1}(\omega)+T_{2}(\omega)-2 T_{3}(\omega)\right],
$$

where

$$
\begin{aligned}
T_{1}(u)= & \cos ^{2}\left(2 \psi_{1}\right) \cdot 4 \sin ^{2}(\beta u)\left[\mu _ { 1 } ^ { 2 } \left(1+\cos ^{2}(u)\right.\right. \\
& \left.-2 \cos (u) \cos \left(u \mu_{1}\right)\right)-2 \mu_{1} \sin (u) \sin \left(u \mu_{1}\right) \\
& \left.+\sin ^{2}(u)\right],
\end{aligned}
$$

$$
\begin{aligned}
T_{2}(u)= & \cos ^{2}\left(2 \psi_{2}\right) \cdot 4 \sin ^{2}(u)\left[\mu _ { 2 } ^ { 2 } \left(1+\cos ^{2}(\beta u)\right.\right. \\
& \left.-2 \cos (\beta u) \cos \left(\beta u \mu_{2}\right)\right) \\
& \left.-2 \mu_{2} \sin (\beta u) \sin \left(\beta u \mu_{2}\right)+\sin ^{2}(\beta u)\right],
\end{aligned}
$$

$$
T_{3}(u)=\cos \left(2 \psi_{1}\right) \cos \left(2 \psi_{2}\right) \cdot 4 \sin (u) \sin (\beta u) \eta(u),
$$

with $u=\omega \tau, \mu_{i}=\cos \theta_{i}$, and where

$$
\begin{aligned}
\eta\left(u, \theta_{1}, \theta_{2}\right)= & {\left[\cos (u)-\cos \left(u \mu_{1}\right)\right][\cos (\beta u)} \\
& \left.-\cos \left(\beta u \mu_{2}\right)\right] \mu_{1} \mu_{2}+\left[\sin (u)-\mu_{1} \sin \left(u \mu_{1}\right)\right] \\
& \times\left[\sin (\beta u)-\mu_{2} \sin \left(\beta u \mu_{2}\right)\right]
\end{aligned}
$$

has been defined for convenience. The propagation angles $\theta_{i}$ and principal polarization angles $\psi_{i}$ are defined with respect to the $i^{\text {th }}$ arm using the geometric conventions of paper I. The expression for the power in the detector, as given by Eq. (13), is a complicated function of frequency and of the orientation between the propagation vector of the gravitational wave and the interferometer, and represents the antenna pattern for the detector.

It is customary to describe the average sensitivity of the instrument by considering the isotropic power, obtained by averaging the antenna pattern over all propagation vectors and all polarizations [13]. Using the definition of $\mathcal{R}(\omega)$ from Eq. (8), with the average isotropic power computed using the geometric averaging procedure of paper I with Eqs. (14)(17), the gravitational wave transfer function is found to be

$$
\begin{aligned}
\mathcal{R}(u)= & \left(\frac{\tau}{u}\right)^{2}\left\{2 \sin ^{2}(\beta u)\left[\left(1+\cos ^{2}(u)\right)\left(\frac{1}{3}-\frac{2}{u^{2}}\right)+\sin ^{2}(u)+\frac{4}{u^{3}} \sin (u) \cos (u)\right]+2 \sin ^{2}(u)\left[\left(1+\cos ^{2}(\beta u)\right)\left(\frac{1}{3}-\frac{2}{(\beta u)^{2}}\right)\right.\right. \\
& \left.\left.+\sin ^{2}(\beta u)+\frac{4}{(\beta u)^{3}} \sin (\beta u) \cos (\beta u)\right]-\frac{1}{\pi} \sin (u) \sin (\beta u) \int_{0}^{2 \pi} d \epsilon \int_{-1}^{+1} d \mu_{1}\left(1-2 \sin ^{2} \alpha\right) \eta\left(u, \theta_{1}, \theta_{2}\right)\right\} .
\end{aligned}
$$




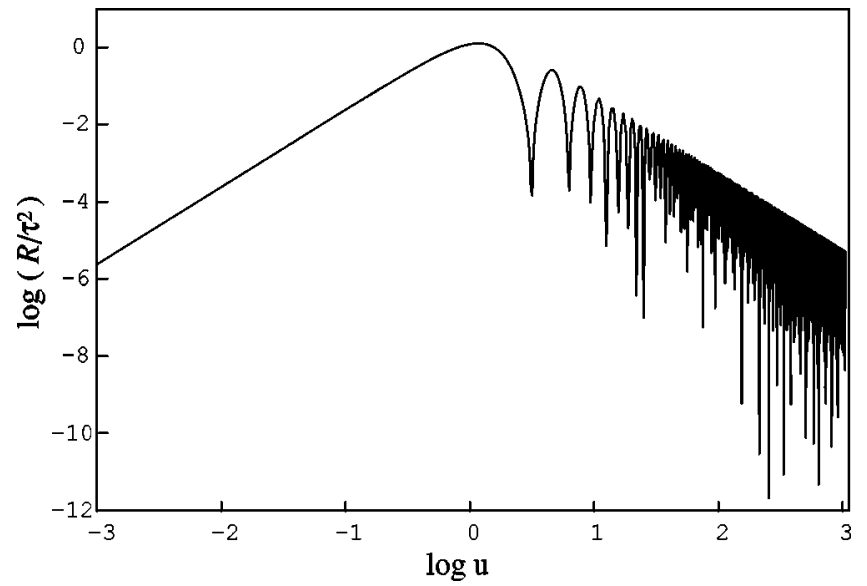

FIG. 2. The dimensionless gravitational wave transfer function, $\mathcal{R} / \tau^{2}$, plotted against the dimensionless frequency parameter $u$ $=\omega \tau$, for a value of $\beta=1.0$. The uneven minima shown in the figure are artifacts of the stepsize of the plot. As can be seen in Eq. (18), the gravitational wave transfer function goes to zero (a minimum in the figure) whenever $u=\pi$ for the $\beta=1.0$ case.

The remaining integral can be evaluated using simple numerical techniques, after relating the angular variables as described in paper I, where

$$
\sin \alpha=\frac{\sin \gamma \sin \epsilon}{\sqrt{1-\mu_{2}^{2}}}
$$

and

$$
\mu_{2}=\mu_{1} \cos \gamma+\sin \gamma \cos \epsilon \sqrt{1-\mu_{1}^{2}}
$$

Here $\gamma$ is the opening angle of the interferometer, and $\epsilon$ is the inclination of the gravitational wave propagation vector to the plane of the interferometer. The complete gravitational wave transfer function is plotted in Fig. 2 for $\beta=1$ ("equal arm") and Fig. 3 for $\beta=0.01$ ("unequal arm") examples.

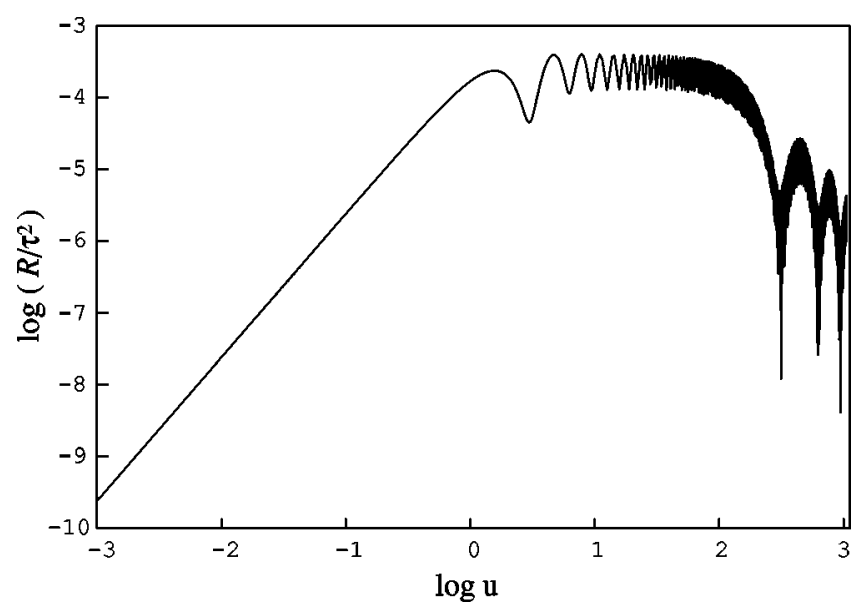

FIG. 3. The dimensionless gravitational wave transfer function $\mathcal{R} / \tau^{2}$ plotted against the dimensionless parameter $u=\omega \tau$, for a value of $\beta=0.01$.
As may be seen in the figure, the low-frequency (small $u$ ) response of the detector to a gravitational wave signal is four orders of magnitude lower for the $\beta=0.01$ detector than for the equal arm detector, implying that the (amplitude) signal will be two orders of magnitude lower - the detected signal level is proportional to the length of the shortest arm. However, once the period of the gravitational wave falls inside the light-time of the longest arm, $u \sim 1$, the equal-arm detector $(\beta=1)$ response begins to fall off while the unequal-arm detector $(\beta=0.01)$ response is roughly flat up to a period corresponding to the light-time in the shortest arm.

The dropoff at low frequencies is a result of the fact that the variable $X(t)$ is formed by subtracting each $z_{i}$ from itself, offset by the light time in the opposite arm. Thus, in the low-frequency limit, the two copies of the signal strongly overlap and the signal is almost entirely subtracted away. For equal arms, the response of the detector is likewise subtracted to zero when an integer number of wavelengths fits in the arm length, as seen in the high-frequency portion of the $\beta=1$ curve. For the unequal-arm case, this does not occur, because the subtraction of two versions of the signal in each arm is done at different light times in the two arms, so whatever period signal cancels in one arm will typically not cancel in the other. However, as may be seen in the $\beta=0.01$ case, the response drops sharply to zero at $\log u \simeq 2.5$ where exactly one wavelength fits into the short arm and exactly one hundred fit into the long arm. The point $\log u \simeq 2.5$ is equivalent to $f \sim 10^{0.5} \mathrm{~Hz}$ for LISA armlength of $c \tau=5$ $\times 10^{9} \mathrm{~m}$ ).

However, the response of the detector's $X(t)$ signal is not the whole story. The ability of a detector to detect a signal depends on both the signal in the detector and on the competing noise. As we shall see in the next section, when the $X(t)$ variable is formed, the noise in each arm is likewise subtracted away in most of the places where the signal is lost (e.g., at low frequency), so the ratio of signal to noise remains high.

\section{Noise transfer function using the $X(t)$ variable}

The noise sources for LISA may be divided into categories in two different ways. First, a noise source may be either one-way (affecting only the incoming or the outgoing signal at a spacecraft, but not both) or two-way (affecting both incoming and outgoing signals at the same time). A one-way noise source will have a transfer function of 2 , since there are 2 spacecraft in each leg contributing equal amounts of such noise [14]. The transfer function for two-way noise sources, however, will be more complicated due to the internal correlation. A single two-way noise fluctuation in the central spacecraft of the interferometer will affect the incoming signal immediately, and then, a round-trip light-time later, will affect the measured signal again in the same way. In the time domain, the effect in the $i^{t h}$ arm of a fluctuation $n(t)$ will be $n_{i}(t)=n(t)+n\left(t-2 \tau_{i}\right)$. The transfer function for this time-delayed sum is $4 \cos ^{2}\left(2 \pi f \tau_{i}\right)$. If an end spacecraft has noise that affects both incoming and outgoing beams, it will affect them at almost the same time, coherently 
and with no delay, giving a transfer function contribution of 4. The noise transfer function for a single arm for a two-way noise source is therefore

$$
4+4 \cos ^{2}\left(2 \pi f \tau_{i}\right) .
$$

Examples of one-way noise are thermal noise in the laser receiver electronics or a mechanical change in the optical pathlength in the outgoing laser signal before it gets to the main telescope optics. Examples of two-way noise are parasitic forces on the accelerometer proof mass or thermal changes in the optical pathlength in the main telescope.

A second way in which noise sources may be classified is by how they scale when there is a change in armlength in the interferometer. The first type of noise in this classification scheme is what we call "position noise," in which the size of the noise in radians of phase is independent of the length of the arm. Accelerometer noise and thermal noise in the laser electronics are examples of position noise. The second type of noise is what we call "strain noise," in which the size of the noise scales with armlength. Examples of strain noise include shot noise and pointing jitter (if it is dominated by low power in the incoming beacon). Position noises may be either one-way or two-way, but we can think of no two-way strain noise sources.

The transfer functions that connect the noise in the instrument to the $X$ variable depend on the type of noise. We begin by considering the noise terms in Eq. (6):

$$
\sigma(t)=n_{1}(t)-n_{2}(t)-\left[n_{1}(t-2 \beta \tau)-n_{2}(t-2 \tau)\right] .
$$

We then go to the frequency domain, squaring and timeaveraging to relate the mean square noise to its power spectrum:

$$
\left\langle\sigma^{2}\right\rangle=\frac{1}{2 \pi} \int d \omega 4\left[\tilde{n}_{1}^{2} \sin ^{2}(\beta u)+\tilde{n}_{2}^{2} \sin ^{2}(u)\right]
$$

where cross terms (e.g., $\tilde{n}_{1} \tilde{n}_{2}$ ) have been neglected under the assumption that noise in the two arms will be independent and uncorrelated. Note that $\tilde{n}_{1}^{2}$ is the power spectrum in the long arm (length $\tau$ ) and $\tilde{n}_{2}^{2}$ is the power spectrum in the short arm (length $\beta \tau$ ).

Since the noise in the detectors includes different types, with different transfer functions, it is not possible to write a single transfer function giving the response of the $X$ variable to noise, so let us consider the various noise categories one at a time. We first consider position noise, for which $\tilde{n}^{2} \equiv \tilde{n}_{1}^{2}$ $=\tilde{n}_{2}^{2}$. Then, using Eq. (23), we find the transfer function for one-way position noise to be

$$
\mathcal{R}_{1}=8\left(\sin ^{2}(\beta u)+\sin ^{2}(u)\right),
$$

where, as we noted above, there is a factor of 2 representing the noise from the two spacecraft in each arm. Two-way position noise must include the transfer function from Eq. (21), giving

$$
\mathcal{R}_{2}=16\left[\sin ^{2}(\beta u)\left(1+\cos ^{2}(u)\right)+\sin ^{2}(u)\left(1+\cos ^{2}(\beta u)\right)\right] .
$$

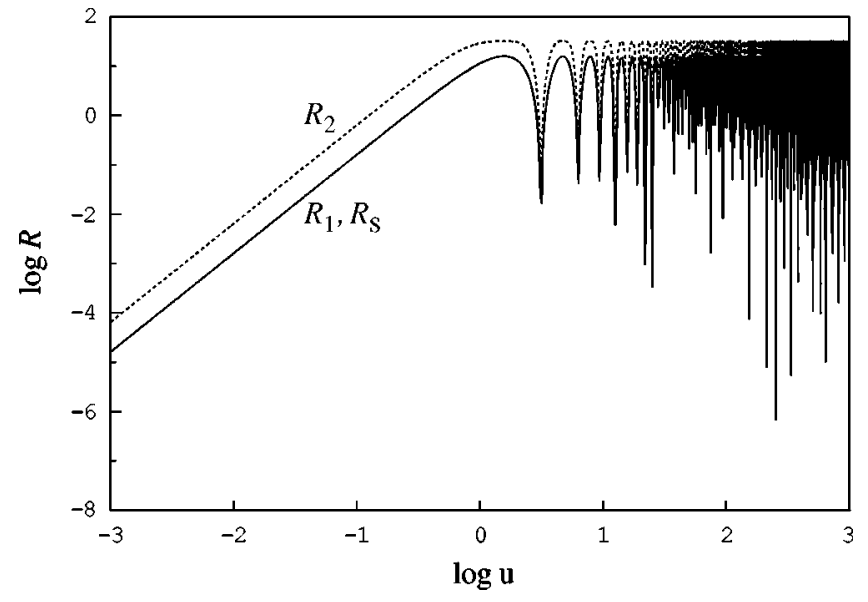

FIG. 4. The noise transfer functions for $\beta=1$ as functions of the dimensionless frequency parameter $u=\omega \tau$. Notice that the transfer function for position noise $\left(\mathcal{R}_{1}\right)$ is identical to the transfer function for shot noise $\left(\mathcal{R}_{s}\right)$ in the $\beta=1$ limit.

Strain noise scales with armlength, and is hence smaller in the shorter arm, so that $\tilde{n}^{2} \equiv \tilde{n}_{1}^{2}=\tilde{n}_{2}^{2} / \beta^{2}$. Its transfer function is therefore

$$
\mathcal{R}_{s}=8\left(\sin ^{2}(\beta u)+\beta^{2} \sin ^{2}(u)\right),
$$

where the factor of 2 for the two spacecraft has again been included.

When $\beta=1$, the transfer functions for strain noise and one-way position noise [Eqs. (24) and (26)] are identical and have zeros at $u_{n}=n \pi$, where $n$ is zero or a positive integer. These are exactly the places where the $\beta=1$ transfer function for gravitational wave signal (Fig. 2) has its zeros. When $\beta<1$, the situation is more complicated. Both $\mathcal{R}_{1}$ and $\mathcal{R}_{s}$ share the $\sin ^{2}(\beta u)$ term which will go to zero at $u=0$ and at multiples of $u=\pi / \beta$. The $\sin ^{2}(u)$ terms in $\mathcal{R}_{1}$ and $\mathcal{R}_{s}$ have their zeros at multiples of the lower frequency, $u=\pi$. In $\mathcal{R}_{1}$, this term will be larger than the $\sin ^{2}(\beta u)$ term at low frequencies, since near $u=0, \sin ^{2}(u) \simeq u^{2}$, while $\sin ^{2}(\beta u) \simeq \beta^{2} u^{2}$. In $\mathcal{R}_{s}$, these terms will be equal in the low-frequency limit,

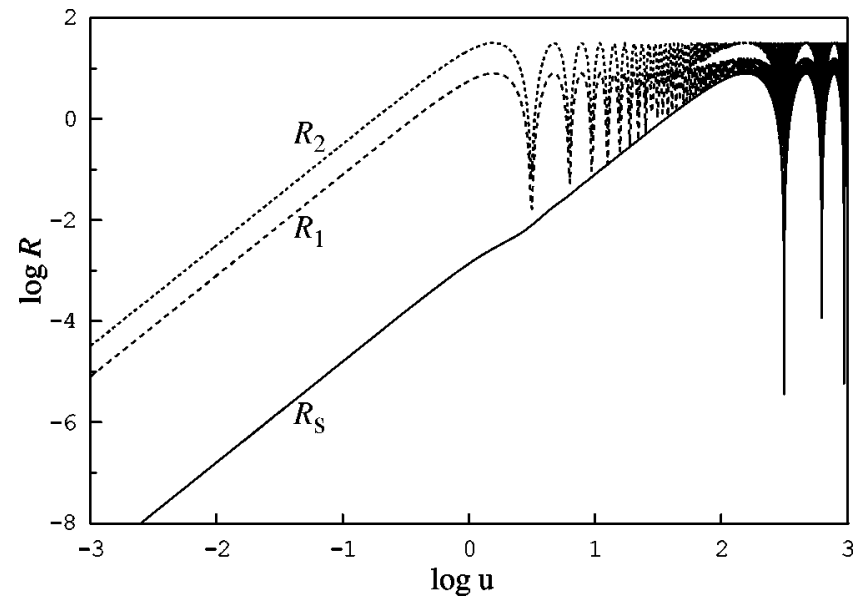

FIG. 5. The noise transfer functions for $\beta=0.01$ as functions of the dimensionless frequency parameter $u=\omega \tau$. 


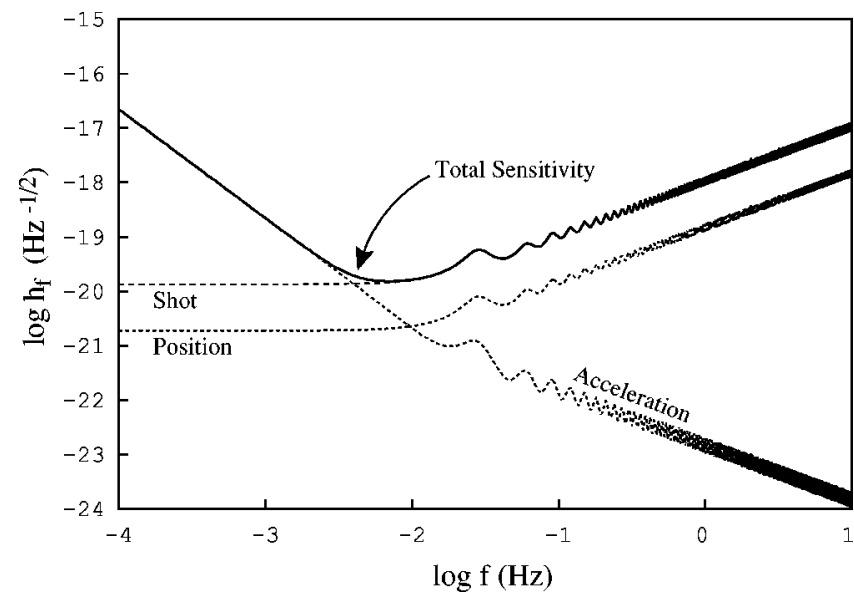

FIG. 6. The sensitivity curve $(S N R=1)$ for $\beta=1$. Overlayed are the sensitivity curves for each of the individual noise spectra (acceleration noise, shot noise, position noise). The noise spectra are taken to be at the LISA target design values, except position noise, which is taken to be $1 / 10$ th the LISA value.

because of the factor $\beta^{2}$ that multiplies the $\sin ^{2}(u)$ term. Thus, in the low frequency limit, the strain noise transfer function will be $2 \beta^{2}$ times the one-way position noise transfer function. When $\beta \ll 1$, the transfer function for one-way position noise will have sharp drops at multiples of $u=\pi$, down to the level of its $\sin ^{2}(\beta u)$ term. These behaviors are shown in Fig. 4 and Fig. 5.

\section{Sensitivity curve}

The signal to noise ratio is the ratio of the signal power in the detector to the noise power in the detector:

$$
\mathrm{SNR}=\frac{S_{h} \mathcal{R}}{S_{s} \mathcal{R}_{s}+S_{1} \mathcal{R}_{1}+S_{2} \mathcal{R}_{2}} .
$$

where $S_{s}, S_{1}$, and $S_{2}$ are the spectra of strain noise and one-way and two-way position noise, respectively, and $\mathcal{R}$ is the gravitational wave transfer function given by Eq. (18).

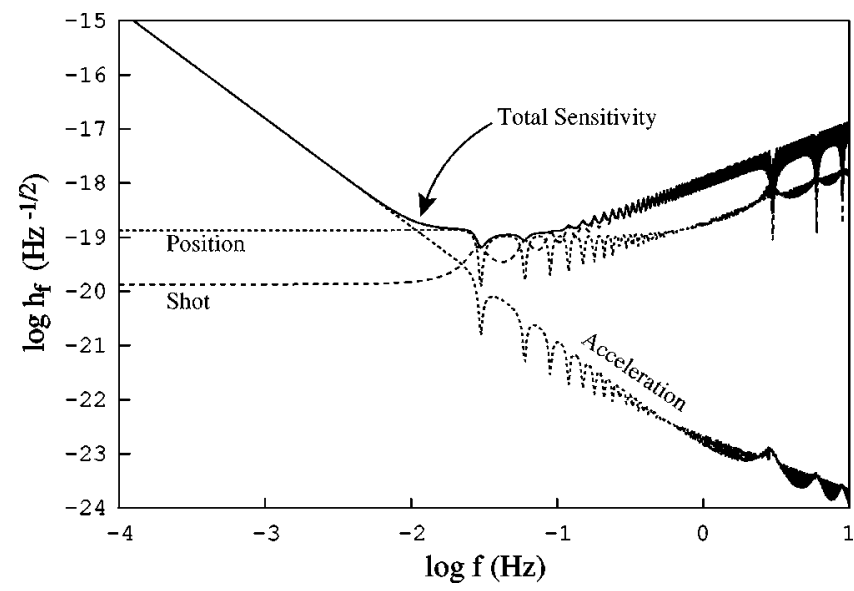

FIG. 7. The sensitivity curve $(S N R=1)$ for $\beta=0.01$. Overlayed are the sensitivity curves for each of the individual noise spectra, as in the previous figure.
Setting SNR $=1$ and solving for $h_{f} \equiv \sqrt{S_{h}}$ yields the instrument sensitivity curve as defined in paper I:

$$
h_{f}=\sqrt{S_{h}}=\sqrt{\frac{S_{s} \mathcal{R}_{s}+S_{1} \mathcal{R}_{1}+S_{2} \mathcal{R}_{2}}{\mathcal{R}}} .
$$

Figures 6 and 7 show the sensitivity curves, computed using Eq. (28), for $\beta=1$ and $\beta=0.01$ respectively. The noise values used are taken to be the LISA target design values (computed as described in paper I). The shot noise and acceleration noise levels are set at the standard LISA values. In addition, a flat one-way position noise spectrum is assumed at $1 / 10^{\text {th }}$ the LISA shot-noise value. Also plotted in Figs. 6 and 7 are sensitivity curves representing each of the three components of the total noise, taken one at a time.

\section{DISCUSSION}

As may be seen in Fig. 7, the low-frequency sensitivity for unequal arms, being set by the two-way position noise in the accelerometer, is degraded over the equal-arm case by the ratio of the two arms. In other words, the sensitivity at lowest frequencies is set by the sensitivity of the shortest arm. At middle and high frequencies, the situation is more complicated. If the dominant noise is strain noise, then the sensitivity is independent of $\beta$ in this frequency range. However, if the dominant noise is position noise, then the sensitivity curve at high frequencies will rise in proportion to $\beta$, though its flat floor will extend to higher frequency, from the $1 /(2 \pi \tau)$ of the equal-arm case to $1 /(2 \pi \beta \tau)$ when the armlength ratio is $\beta$.

The implications of these results for mission design are obvious. If the armlengths are not equal, the low-frequency sensitivity is degraded by a factor $1 / \beta$, the ratio of the armlengths. If the high-frequency noise can be guaranteed to be strain noise, even in the shorter arm, then the high-frequency sensitivity is unaffected by the unequal arms. If the noise at high frequency is dominated by position noise, then the high frequency sensitivity is degraded by the factor $1 / \beta$, but the sensitivity remains flat up to a frequency $1 /(2 \pi \beta \tau)$, where it turns over and joins the strain noise curve. Thus, as long as the position-noise sources can be kept well below the shot noise and other strain-noise contributions, a change in armlength ratio from strict equality will not degrade the highfrequency portion of the sensitivity curves. However, as the length of one of the arms is shortened, small position noise sources will become important and eventually dominate.

Let us consider the example of Schutz's 4-spacecraft configuration (Fig. 1). Since this configuration will have $\beta$ $=0.5$, the low-frequency sensitivity curve will be a factor of 2 higher (hence less sensitive). The current error budget for LISA assumes that the high-frequency portion of the window is dominated by position noise approximately three times the shot noise. If this remains the case, then the high-frequency section of the curve will likewise be a factor of 2 higher up to a frequency twice as high as the LISA sensitivity "knee" at $f=1 /(2 \pi \tau)$, at which point it would turn up and join the current LISA high-frequency ramp. The shot noise is determined by the power of the laser and by the size and effi- 
ciency of the optics, and there is nothing beyond brute-force improvements in these parameters that will lower the shot noise. The contributions to position noise, on the other hand, are due to optics quality, the attitude control system, Brownian noise in the electronics, thermal noise in the optical path length, etc. These are more complex and are amenable to reduction by careful or innovative engineering design. If these noise sources can be reduced to a fraction of the shot noise, not only will the LISA noise floor be reduced by a factor of 4 , but the Schutz modification will have highfrequency performance that is undiminished by the reduction of the length of one arm.

Finally, we describe a totally unfeasible mission design that is nevertheless interesting for instructive purposes. Let us consider a two-spacecraft "interferometer," where one of the spacecraft contains a fiber optic delay line, of length 5 $\mathrm{km}$, that acts as the second arm of the interferometer. If the distance between the two spacecraft is $5 \times 10^{6} \mathrm{~km}$, we will have $\beta=10^{-6}$. The use of the $X(t)$ variable will eliminate laser phase noise, exactly as it does in arms that are more nearly equal. A rigidly attached reflector at the far end of the fiber-optic line would eliminate accelerometer noise, but, of course, would replace it with thermal fluctuation in the optical path length in the fiber. However, a concatenation of fibers with well-chosen thermal pathlength coefficients could produce a fiber tuned to have a coefficient very near zero. This, combined with multilevel thermal isolation, could keep this noise source very small. The key to the sensitivity of this configuration is the position noise. If a way could be found to reduce position noise to less than $10^{-6}$ of the LISA shot noise, then this two-spacecraft interferometer would have the same sensitivity as a conventional three-spacecraft interferometer.

\section{ACKNOWLEDGMENTS}

S.L.L. acknowledges support for this work under LISA contract number PO 1217163, and the NASA EPSCoR Program through Cooperative Agreement NCC5-410. The work of W.A.H. was supported in part by NSF Grant No. PHY0098787 and the NASA EPSCoR Program through Cooperative Agreement NCC5-579. R.W.H. was supported by NASA grant NAGS5-11469 and NCC5-579.
[1] P. Bender, et al., LISA Pre-Phase A Report (second ed.) (1998) (unpublished).

[2] M. Tinto and J.W. Armstrong, Phys. Rev. D 59, 102003 (1999).

[3] R.W. Hellings, Phys. Rev. D 64, 022002 (2001).

[4] J.W. Armstrong, F.B. Estabrook, and M. Tinto, Astrophys. J. 527, 814 (1999).

[5] S.L. Larson, W.A. Hiscock, and R.W. Hellings, Phys. Rev. D 62, 062001 (2000).

[6] R. Schilling, Class. Quantum Grav. 14, 1513 (1997).

[7] F.B. Estabrook, M. Tinto, and J.W. Armstrong, Phys. Rev. D 62, 042002 (2000)

[8] M. Tinto, F.B. Estabrook, and J.W. Armstrong, Phys. Rev. D 65, 082003 (2002).

[9] B. Schutz, Class. Quantum Grav. 18, 4145 (2001).

[10] F.B. Estabrook and H.D. Wahlquist, Gen. Relativ. Gravit. 6, 439 (1975).

[11] This form of $X(t)$ assumes that the lasers in the end spacecraft are phase-locked to the signals they receive from the central spacecraft. There is a form for $X(t)$ that does not make this assumption and that can thereby be converted to interferometers centered on the other spacecraft in the constellation. However, the sensitivity we derive using this form will be valid for the more general form as well, and will therefore apply to signals formed with any spacecraft as vertex.

[12] In paper I, the signal part of $X(t)$ was called $\Xi(t)$ in the limit where $\tau_{1} \rightarrow \tau_{2}$.

[13] The input gravitational wave state given in Eq. (1) is linearly polarized. As in paper I, the averaging procedure over all linearly polarized states produces the same response function as averaging over a more general elliptically polarized state with an appropriately weighted distribution.

[14] This choice of putting the factor of 2 into the transfer function differs from our convention in paper I, where such factors were included in the noise spectra. We have found it clearer to define the transfer function as the one that gives the noise power spectrum in $X$ as $S_{X}=R S_{N}$, where $S_{N}$ is the noise spectral density of a single type in a single spacecraft. 